\title{
REACH-2: first biomarker-based anti-angiogenic therapy in patients with advanced hepatocellular carcinoma
}

\author{
Hong Jae Chon ${ }^{1,2}$, Chan Kim ${ }^{1,2}$ \\ ${ }^{1}$ Medical Oncology, CHA Bundang Medical Center, CHA University, Seongnam, Republic of Korea; ${ }^{2}$ Laboratory of Translational Immuno- \\ Oncology, CHA University, Seongnam, Republic of Korea \\ Correspondence to: Chan Kim, MD, PhD. Medical Oncology, CHA Bundang Medical Center, CHA University, 59 Yatap-ro, Bundang-gu, Seongnam \\ 13496, Republic of Korea. Email: chan@cha.ac.kr or larrel80@gmail.com. \\ Provenance and Peer Review: This article was commissioned by the editorial office, Chinese Clinical Oncology. The article did not undergo external peer review. \\ Comment on: Zhu AX, Kang YK, Yen CJ, et al. Ramucirumab after sorafenib in patients with advanced hepatocellular carcinoma and increased \\ $\alpha$-fetoprotein concentrations (REACH-2): a randomised, double-blind, placebo-controlled, phase 3 trial. Lancet Oncol 2019;20:282-96.
}

Submitted Dec 21, 2019. Accepted for publication Jan 06, 2020.

doi: $10.21037 /$ cco.2020.01.04

View this article at: http://dx.doi.org/10.21037/cco.2020.01.04

Hepatocellular carcinoma (HCC) is known as a hypervascular tumor $(1,2)$. Various angiogenic pathways are known to be deregulated and involved in HCC; diverse proangiogenic and anti-angiogenic factors establish complex networks to regulate tumor vasculature in $\operatorname{HCC}(2,3)$. Therefore, multiple anti-angiogenic therapies have been developed and approved to treat patients with advanced HCC over the last decade $(1,4-8)$. Because the efficacy of anti-angiogenic therapies was modest and transient, there has been an intense effort to discover biomarkers that can select patients with more favorable responses, thereby optimizing the clinical benefit of anti-angiogenic therapy in HCC (9-12).

Recently, Zhu et al. suggested biomarker-based antiangiogenic therapy in patients with HCC after sorafenib based on the results of a pivotal REACH-2 trial (8). This is the first positive trial with biomarker-based patient selection to open the personalized medicine era of advanced HCC (13).

The REACH-2 trial is a randomized, double-blind, phase III clinical trial comparing ramucirumab, an antiVEGFR2 monoclonal antibody, versus placebo as a secondline treatment after first-line sorafenib treatment in patients with advanced HCC. In REACH-2 trial, patients with a baseline alpha-fetoprotein (AFP) $\geq 400 \mathrm{ng} / \mathrm{mL}$ were included based on the results of previous phase III REACH trial in which patients with high baseline AFP showed improvements in overall survival (OS) (14). All patients were treated with first-line sorafenib and Child-Pugh class
A. Stratification factors were geographic region, ECOG performance status, and macrovasclar invasion. A total of 292 patients were randomized to either ramucirumab $(\mathrm{n}=197)$ or placebo group $(\mathrm{n}=95)$ in a 2:1 ratio. The primary endpoint of the trial was OS and the secondary endpoints were progression-free survival (PFS), time-to-progression (TTP), objective response rate (ORR), and safety profiles. Patients in the ramucirumab group were treated with intravenous injections of ramucirumab $(8 \mathrm{mg} / \mathrm{kg})$ on day 1 of 14-day cycle. Ramucirumab showed superior OS (median 8.5 vs. 7.3 months; HR 0.710; $\mathrm{P}=0.0199$ ) and PFS (median 2.8 vs. 1.6 months; HR 0.452; $\mathrm{P}<0.001$ ) (Table 1). Although disease control rate was significantly higher in the ramucirumab group $(59.9 \%$ vs. $38.9 \%, \mathrm{P}=0.0006)$, there were no significant changes in ORR ( $5 \%$ vs. $1 \% ; \mathrm{P}=0.1697)$, indicating ramucirumab, like other anti-angiogenic agents, is more suited to tumor stabilization than regression of HCC. Safety profiles showed consistent results with previous trials of ramucirumab. The most common adverse events related to ramucirumab were fatigue (27\%), followed by peripheral edema $(25 \%)$ and hypertension $(25 \%)$. Overall, this study met its primary endpoint and demonstrated the efficacy of ramucirumab in patients with advanced HCC and AFP $\geq 400 \mathrm{ng} / \mathrm{mL}$ who had been previously treated with sorafenib. The pooled analysis of REACH (only patients with AFP $\geq 400 \mathrm{ng} / \mathrm{mL}$ ) and REACH-2 trials also revealed consistent results (Table 1). When a total of 542 patients were analyzed, ramucirumab 
Table 1 Summary of REACH-2 and pooled REACH/REACH-2 (AFP $\geq 400 \mathrm{ng} / \mathrm{mL}$ )

\begin{tabular}{lcccc}
\hline & \multicolumn{2}{c}{ REACH-2 } & & Pooled REACH and REACH-2 (AFP $\geq 400 \mathrm{ng} / \mathrm{mL})$ \\
\cline { 2 - 3 } Median OS & $8.5(7.0-10.6)$ & $7.3(5.4-9.1)$ & Ramucirumab $(\mathrm{n}=316)$ & Placebo $(\mathrm{n}=226)$ \\
HR for OS $(95 \% \mathrm{Cl}), \mathrm{P}$ & $0.710(0.531-0.949), 0.0199$ & $8.1(6.9-9.3)$ & $0.694(0.571-0.842), 0.0002$ \\
Median PFS & $2.8(2.8-4.1)$ & $1.6(1.5-2.7)$ & $2.8(2.7-2.8)$ & $0.572(0.472-0.694),<0.0001$ \\
HR for PFS, P & $0.452(0.339-0.603),<0.0001$ & $(1.5-2.0)$ \\
\hline
\end{tabular}

AFP, alpha-fetoprotein; OS, overall survival; PFS, progression-free survival.

treatment significantly increased OS by 3.1 months (median 8.1 vs. 5.0 months; $\mathrm{HR}=0.694, \mathrm{P}=0.002$ ). Moreover, it can improve ORR (5.4\% vs. $0.9 \%$; $\mathrm{P}=0.004)$ and PFS (median 2.8 vs. 1.5 months; $\mathrm{P}<0.0001$ ).

Ramucirumab is a fully human monoclonal antibody against VEGFR2 and, thus, is expected to more selectively block VEGF/VEGFR2 signaling with less off-target effects than other multi-target anti-angiogenic tyrosine kinase inhibitors (TKIs) $(8,15)$. In REACH-2 trial ramucirumab was well tolerated and the only grade $\geq 3$ adverse events that occurred in $>5 \%$ of patients in ramucirumab group were hypertension and hyponatremia. Therefore, the duration of ramucirumab treatment was almost the same as the duration of PFS without significant interruptions. In contrast, oral VEGFR TKIs induce chronic toxicities, such as hand-foot syndrome and diarrhea, that result in dose reductions, treatment delays or discontinuation. Therefore, ramucirumab could be a better treatment option to patients who cannot tolerate oral VEGFR TKIs. And this could be especially true for East Asian patients with advanced HCC because they are known to be more susceptible to oral TKIs and have more frequent TKI-related toxicities, such as hand-foot syndrome, compared to patients of European ancestry (16).

AFP is a well-defined tumor marker in patients with HCC and approximately $50 \%$ of HCC patients have elevated baseline AFP ( $\geq 400 \mathrm{ng} / \mathrm{mL}$ ) (17). The prognostic role of AFP has been evaluated in many prospective studies and a high AFP level at baseline was consistently associated with poor prognosis in patients with HCC (13). However, its biologic functions, especially regarding tumor angiogenesis, have not been well elucidated. Few studies have reported that AFP itself may act as a pro-angiogenic factor in a VEGF-dependent manner to promote robust angiogenesis within tumors (18-20). Silencing of AFP markedly reduces the amount of VEGF secreted from
HCC cells (17). Therefore, AFP may stimulate HCC cells to produce VEGF, which can promote tumor angiogenesis and establish a VEGF-rich tumor microenvironment that contributes to the aggressive phenotype of HCC. In supporting this hypothesis, elevated AFP levels correlated with high expression of VEGF-A, as well as its receptor, VEGFR2, in the tumor tissues of patients with HCC $(21,22)$. However, because preclinical and clinical evidence is very limited at present, it is hard to conclude whether AFP itself is a direct proangiogenic factor that establish highly-angiogenic HCC or AFP is just an indirect surrogate marker for highly aggressive subtypes of HCC. Therefore, the biological role of AFP during HCC carcinogenesis and the characteristics of AFP-high HCCs need to be thoroughly elucidated in further studies.

In conclusion, Zhu et al. demonstrated that AFP-based patient selection is a valid strategy in patients with advanced HCC and ramucirumab will be a well-tolerated secondline therapy after sorafenib failure when baseline AFP $\geq 400 \mathrm{ng} / \mathrm{mL}$.

\section{Acknowledgments}

Funding: None.

\section{Footnote}

Conflicts of Interest: Both authors have completed the ICMJE uniform disclosure form (available at http://dx.doi. org/10.21037/cco.2020.01.04). Dr. Chon has received consultancy and advisory fees from Bayer, Eisai, ONO, and MSD. The other author has no conflicts of interest to declare.

Ethical Statement: The authors are accountable for all aspects of the work in ensuring that questions related 
to the accuracy or integrity of any part of the work are appropriately investigated and resolved.

Open Access Statement: This is an Open Access article distributed in accordance with the Creative Commons Attribution-NonCommercial-NoDerivs 4.0 International License (CC BY-NC-ND 4.0), which permits the noncommercial replication and distribution of the article with the strict proviso that no changes or edits are made and the original work is properly cited (including links to both the formal publication through the relevant DOI and the license). See: https://creativecommons.org/licenses/by-nc-nd/4.0/.

\section{References}

1. Roviello G, Sohbani N, Petrioli R, et al. Ramucirumab as a second line therapy for advanced HCC: a significant achievement or a wasted opportunity for personalised therapy? Invest New Drugs 2019;37:1274-88.

2. Morse MA, Sun W, Kim R, et al. The role of angiogenesis in hepatocellular carcinoma. Clin Cancer Res 2019;25:91220.

3. Mossenta M, Busato D, Baboci L, et al. New Insight into Therapies Targeting Angiogenesis in Hepatocellular Carcinoma. Cancers (Basel) 2019. doi: 10.3390/ cancers 11081086.

4. Llovet JM, Ricci S, Mazzaferro V, et al. Sorafenib in advanced hepatocellular carcinoma. N Engl J Med 2008;359:378-90.

5. Bruix J, Qin S, Merle P, et al. Regorafenib for patients with hepatocellular carcinoma who progressed on sorafenib treatment (RESORCE): a randomised, double-blind, placebo-controlled, phase 3 trial. Lancet 2017;389:56-66.

6. Abou-Alfa GK, Meyer T, Cheng AL, et al. Cabozantinib in patients with advanced and progressing hepatocellular carcinoma. N Engl J Med 2018;379:54-63.

7. Kudo M, Finn RS, Qin S, et al. Lenvatinib versus sorafenib in first-line treatment of patients with unresectable hepatocellular carcinoma: a randomised phase 3 noninferiority trial. Lancet 2018;391:1163-73.

8. Zhu AX, Kang YK, Yen CJ, et al. Ramucirumab after sorafenib in patients with advanced hepatocellular carcinoma and increased $\alpha$-fetoprotein concentrations (REACH-2): a randomised, double-blind, placebocontrolled, phase 3 trial. Lancet Oncol 2019;20:282-96.

9. Kim C, Yang H, Fukushima Y, et al. Vascular RhoJ is an effective and selective target for tumor angiogenesis and vascular disruption. Cancer Cell 2014;25:102-17.

10. Shao YY, Hsu CH, Cheng AL. Predictive biomarkers of antiangiogenic therapy for advanced hepatocellular carcinoma: where are we? Liver Cancer 2013;2:93-107.

11. Zhu AX, Duda DG, Sahani DV, et al. HCC and angiogenesis: possible targets and future directions. Nat Rev Clin Oncol 2011;8:292-301.

12. Lee JE, Kim C, Yang H, et al. Novel glycosylated VEGF decoy receptor fusion protein, VEGF-Grab, efficiently suppresses tumor angiogenesis and progression. Mol Cancer Ther 2015;14:470-9.

13. Zhu AX, Finn RS, Galle PR, et al. Ramucirumab in advanced hepatocellular carcinoma in REACH-2: the true value of $\alpha$-fetoprotein. Lancet Oncol 2019;20:e191.

14. Zhu AX, Park JO, Ryoo BY, et al. Ramucirumab versus placebo as second-line treatment in patients with advanced hepatocellular carcinoma following first-line therapy with sorafenib (REACH): a randomised, double-blind, multicentre, phase 3 trial. Lancet Oncol 2015;16:859-70.

15. Javle M, Smyth EC, Chau I. Ramucirumab: successfully targeting angiogenesis in gastric cancer. Clin Cancer Res 2014;20:5875-81.

16. Touma JA, McLachlan AJ, Gross AS. The role of ethnicity in personalized dosing of small molecule tyrosine kinase inhibitors used in oncology. Transl Cancer Res 2017;6:S1558-91.

17. Meng W, Li X, Bai Z, et al. Silencing alphafetoprotein inhibits VEGF and MMP-2/9 production in human hepatocellular carcinoma cell. PLoS One 2014;9:e90660.

18. Takahashi Y, Ohta T, Mai M. Angiogenesis of AFP producing gastric carcinoma: correlation with frequent liver metastasis and its inhibition by anti-AFP antibody. Oncol Rep 2004;11:809-13.

19. Liang OD, Korff T, Eckhardt J, et al. Oncodevelopmental $\alpha$-fetoprotein acts as a selective proangiogenic factor on endothelial cell from the fetomaternal unit. J Clin Endocrinol Metab 2004;89:1415-22.

20. Guo RP, Zhong C, Shi M, et al. Clinical value of apoptosis and angiogenesis factors in estimating the prognosis of hepatocellular carcinoma. J Cancer Res Clin Oncol 2006;132:547-55.

21. Ginanni Corradini S, Morini S, Liguori F, et al. Differential vascular endothelial growth factor A protein expression between small hepatocellular carcinoma and 
cirrhosis correlates with serum vascular endothelial growth factor A and alpha-fetoprotein. Liver International 2009;29:103-12.

Cite this article as: Chon HJ, Kim C. REACH-2: first biomarker-based anti-angiogenic therapy in patients with advanced hepatocellular carcinoma. Chin Clin Oncol 2020;9(4):58. doi: 10.21037/cco.2020.01.04
22. Huang J, Zhang X, Tang Q, et al. Prognostic significance and potential therapeutic target of VEGFR2 in hepatocellular carcinoma. J Clin Pathol 2011;64:343-8. 